\title{
Solar overshoot region and small-scale dynamo with realistic energy flux
}

\author{
H. Hotta \\ Department of Physics, Graduate School of Science, Chiba university, 1-33 Yayoi-cho, \\ Inage-ku, Chiba, 263-8522, Japan \\ hotta@chiba-u.jp
}

Received __; accepted _ 


\begin{abstract}
We carry out high resolution calculations of the solar overshoot region with unprecedentedly realistic parameters, especially the small energy flux compared with $\rho c_{\mathrm{s}}^{3}$, where $\rho$ and $c_{\mathrm{s}}$ are density and speed of sound. Our main purpose is to investigate behavior of the overshoot and the small-scale dynamo with parameters as close as possible to thoes of the Sun. Our calculations show that the bottom part of the convection zone becomes subadiabatic, which efficiently suppresses downflows. As a result we see a steep transition from the CZ to the RZ whose width is estimated $0.4 \%$ of the local pressure scale height. This result is consistent with a semi-analytic convection/overshoot model. We also find that the small-scale dynamo becomes efficient with a smaller energy flux. The sudden suppression of the downflows around the base of the convection zone increases the efficiency of the small-scale dynamo.
\end{abstract}

Subject headings: Sun: interior — Sun: dynamo — Stars: interiors

\title{
1. Introduction
}

The Sun is composed of a convectively stable radiative zone (RZ: inner $70 \%$ of radius) and an unstable convection zone (CZ: outer 30\%). Between these two regions, there is thought to be overshoot region (OS). Although a proper description of the OS is crucial to understand the solar rotation profile, especially the tachocline, and the global solar dynamo (e.g. Parker 1993; Brun et al. 2011), there has been a substantial discrepancy between its observations, and what is expected based on analytical approachs, and numerical calculations.

On the observational side, helioseismology uses acoustic mode, i.e., the sound wave. The 
discontinuity of the temperature gradient introduces a characteristic oscillatory component in the frequencies. Thus the helioseismology essentially estimates the temperature gradient to see the structure of the OS. Since the CZ is slightly superadiabatic and the RZ is strongly subadiabatic, this transition can be detected. Basu (1997) sets an upper limit on the thickness of the $\mathrm{OS}\left(d_{\mathrm{os}}\right)$ of $0.05 H_{p}$, where $H_{p} \sim 6 \times 10^{9} \mathrm{~cm}$ is the local pressure scale height at the base of the CZ. Other studies using helioseismology also show similar thin OS (Monteiro et al. 1994; Roxburgh \& Vorontsov 1994; Basu et al. 1994; Basu 2016).

On the analytic side, early simplified models of the OS show results of the rather thick OS of $d_{\mathrm{os}}=(0.2-0.4) H_{p}$ (van Ballegooijen 1982; Schmitt et al. 1984; Pidatella \& Stix 1986). These three models used different assumptions, i.e., a non-local mixing length model, overturning convective rolls, and a model of the downflow plumes, respectively. The models produced consistent result, independently of the different assumptions adopted.

On the numerical side, a lot of numerical calculations have been performed. Twoand three-dimensional numerical calculations show thicker OS of $d_{\mathrm{os}}=(0.4-2) H_{p}$ (Roxburgh \& Simmons 1993; Hurlburt et al. 1994; Singh et al. 1995, 1998; Brummell et al. 2002). Rempel (2004) points out that the discrepancy between the simplified and numerical models is caused by the difference of the imposed energy flux from the bottom of the calculations - here the semi-analytic model of Rempel (2004) also shows the thickness of the OS of $0.4 H_{p}$ with a sharp transition region to the RZ. The reason for the high energy flux in the simulations is in order to avoid the severe constraint by the CFL condition when the correct flux is used, however the large energy flux increases the convection velocity and the overshoot penetration depth (see the detailed explanation in $\$ 1.1$ ).

Recent high resolution calculations show that the base of the $\mathrm{CZ}$ is important for both the small- and large-scale dynamo (Hotta et al. 2015a, 2016). Hotta et al. (2015a) show that small-scale magnetic field reaches the equipartition level of the turbulent flow in the 
bulk of the CZ, when the resolution is high enough to resolve turbulent inertia scale well. Especially at the base of the CZ, the strong magnetic field suppresses the small-scale flow and the turbulent RMS velocity is decreased by a factor of two. The magnetic energy there exceeds $90 \%$ of the kinetic energy. Hotta et al. (2016) find that the strong small-scale magnetic field at the base of CZ has a significant effect on the large-scale dynamo. The strong small-scale magnetic field suppresses the small-scale flows which otherwise tends to destroy large-scale features. Thus this suppression of the small-scale flow allows large-scale magnetic field to be generated even with large Reynolds numbers. Both of the above papers, however, investigate only the CZ, i.e., the RZ is excluded. Thus the overshoot region is explicitly ignored in these two papers. We need to improve our understanding of the OS for both the small- and large-scale dynamos.

Other three-dimensional models including the RZ argue that the OS is an important place for the large-scale dynamo in terms of the storage of the large-scale magnetic flux (Browning et al. 2006; Masada et al. 2013; Guerrero et al. 2016). These studies typically adopt large energy flux or softer RZ (smaller absolute value of the superadiabaticity) to avoid numerical difficulties discussed in the next subsection. The values in typical calculations are shown in the next section. These studies thus likely overestimate the thickness of the OS and its ability to store large-scale magnetic flux. In this paper, we address this issue by adopting more realistic solar parameters, such as the imposed energy flux. 


\subsection{The difficulty of including a realistic radiation zone}

In order to discuss the thermal convective stability, a non-dimensional number, superadiabaticity, is useful. The superadiabaticity $(\delta)$ is defined as:

$$
\delta=\nabla-\nabla_{\mathrm{ad}}=-\frac{H_{p}}{c_{\mathrm{p}}} \frac{d s}{d r},
$$

where $\nabla=d \ln T / d \ln p$ is the temperature gradient and $\nabla_{\mathrm{ad}}$ is the adiabatic one. $r, T, p, s$, $c_{\mathrm{p}}$, and $H_{p}=(d \ln p / d r)^{-1}$ are the local radius, temperature, pressure, specific entropy, heat capacity at constant pressure, and pressure scale height, respectively. The estimated values in the solar $\mathrm{CZ}$ and $\mathrm{RZ}$ are $\delta \sim 10^{-7}$ and $-10^{-1}$, respectively (Christensen-Dalsgaard et al. 1996). The superadiabaticity in the $\mathrm{CZ}$ is related to the ratio of the convection velocity $\left(v_{\mathrm{c}}\right)$ and the sound speed $\left(c_{\mathrm{s}}\right)$, i.e., $\delta \sim\left(v_{\mathrm{c}} / c_{\mathrm{s}}\right)^{2}$ using mixing length theory (Vitense 1953). Thus the small superadiabaticity implies that the sound speed is much faster than the convective velocities. This severely constrains the CFL condition and requires a very small time step in terms of the thermal convection is required. In recent numerical calculations, this difficulty is avoided either by using the anelastic approximation (e.g. Miesch et al. 2000) or the reduced speed of sound technique (RSST: Hotta et al. 2012, 2015b) where the propagation of the sound wave is changed.

The small superadiabaticity in the solar $\mathrm{CZ}$ is the consequence of the relatively small imposed energy flux. The energy flux in the CZ can be roughly estimated as $F_{\odot} \sim \rho v_{\mathrm{c}}^{3}$, where $\rho$ is the density. Thus, the superadiabaticity can be expressed as a normalized flux $\delta \sim\left(v_{\mathrm{c}} / c_{\mathrm{s}}\right)^{2} \sim \bar{F}_{\odot}^{2 / 3}$, where the normalized energy flux is expressed with $\bar{F}_{\odot}=F_{\odot} /\left(\rho c_{\mathrm{s}}^{3}\right)$, which is estimated to be $\bar{F}_{\odot} \sim 5 \times 10^{-11}$ at the base of the solar CZ with Model S (Christensen-Dalsgaard et al. 1996).

In the RZ, internal gravity waves oscillate with the Brunt-Väisälä frequency. The 
Brunt-Väisälä frequency $(N)$ is

$$
N^{2}=\frac{g}{c_{\mathrm{p}}} \frac{d s}{d r}=-\frac{1}{\gamma} \frac{c_{\mathrm{s}}^{2}}{H_{p}^{2}} \delta,
$$

where $g$ is the gravitational acceleration. The square root of the absolute value of the superadiabaticity in the $\mathrm{RZ}$ is the ratio of the time scales of the internal gravity wave $(1 / N)$ and the sound wave $\left(H_{p} / c_{\mathrm{s}}\right)$. Since the square root of the superadiabaticity in the solar RZ is close to unity, the time scale for the internal gravity wave and the sound wave is similar in the solar RZ. When the vertical wave length is close to the pressure scale height $\left(\sim H_{p}\right)$, the phase velocity of the internal gravity wave $\left(N \lambda_{\mathrm{v}} /(2 \pi) \sim c_{\mathrm{s}} \sqrt{-\delta / \gamma}\right.$, where $\lambda_{\mathrm{v}}$ is the vertical wave length) can be comparable to the speed of sound. Thus internal gravity waves also require a time step which is very small in terms of the thermal convection. This cannot be avoided with either the anelastic approximation or the RSST.

The essential difficulties to numerically investigate the OS are summarized as:

1. The speed of sound is much faster than the convection velocity in the CZ due to the small energy flux $\left(\bar{F}_{\odot} \sim 5 \times 10^{-11}\right)$.

2. The internal gravity wave has a similar time scale (Brunt-Väisälä frequency) to the sound wave in the RZ.

Additional numerical complications arise because of the significant difference of the superadiabaticities in the $\mathrm{CZ}$ and $\mathrm{RZ}$, which leads to the expectation that the OS or the transition region to the RZ should be very thin. This requires the use of a large number of grid points to resolve this thin layer. When the anelastic approximation is used, the numerical calculation is likely to use softer RZ. For example, Miesch et al. (2000) adopt $\delta \sim-10^{-4}$ in the RZ (see Fig. 1 of Miesch et al. (2000)). Most compressible calculations are done using a very large energy flux of $\bar{F}_{\odot} \sim 10^{-3}$ to $10^{-4}$ (Hurlburt et al. 1994; 
Brummell et al. 2002; Käpylä et al. 2010). This likely overestimates the thickness of the OS.

The purposes of the present study are to:

1. Investigate the dependence of the overshoot depth on the energy flux. Although a calculation with the real solar value $\left(\bar{F}_{\odot} \sim 5 \times 10^{-11}\right)$ is still difficult to carry out, we achieve unprecedentedly small energy flux $\bar{F}_{0}=5 \times 10^{-7}$. We find a scaling law for the normalized energy flux and overshoot depth and estimate a real solar overshoot depth.

2. Fill the gap between the observations and the simplified models. Our calculations includes almost all physics with most realistic parameters of the $\mathrm{CZ}$, the RZ, and the OS. This possibly gives an insight into the realistic solar overshoot region.

3. Compare overshoot depths with and without the magnetic field. Our previous calculation revealed that the small-scale dynamo is very efficient in the solar CZ (Hotta et al. 2015a). The magnetic field in high-resolution calculations possibly influences the behavior of the overshoot layer.

4. Investigate the dependence of the small-scale dynamo on the imposed energy flux, i.e., the superadiabaticity in the CZ.

5. Explore appropriate boundary conditions for the magnetic field at the base of the CZ. Although currently the anelastic approximation and the RSST are not useful for investigations of the OS, these method are necessary in order to cover the time scale of large-scale dynamo. We need to confirm appropriate boundary condition for the important place, the base of the $\mathrm{CZ}$. 


\section{Model}

We solve the three-dimensional magnetohydrodynamic (MHD) equations in a local Cartesian geometry $(x, y, z)$. We define the $x$-direction as that of gravity. The $y$ - and $z$-directions are horizontal directions. The MHD equations with the gravity and radiative heating and cooling are:

$$
\begin{aligned}
\frac{\partial \rho}{\partial t} & =-\nabla \cdot(\rho \mathbf{v}), \\
\frac{\partial}{\partial t}(\rho \mathbf{v}) & =-\nabla \cdot\left[\rho \mathbf{v} \mathbf{v}+\left(p \mathbf{I}+\frac{B^{2}}{8 \pi} \mathbf{I}-\frac{\mathbf{B B}}{4 \pi}\right)\right]-\rho g \mathbf{e}_{x}, \\
\frac{\partial \mathbf{B}}{\partial t} & =\nabla \times(\mathbf{v} \times \mathbf{B}), \\
\frac{\partial E_{\text {total }}}{\partial t} & =-\nabla \cdot\left[\left(E_{\text {total }}+p+\frac{B^{2}}{8 \pi}\right) \mathbf{v}-\frac{(\mathbf{v} \cdot \mathbf{B}) \mathbf{B}}{4 \pi}-\kappa_{\mathrm{r}} \nabla T\right]-\rho v_{x} g+\Gamma, \\
E_{\text {total }} & =\frac{p}{\gamma-1}+\frac{1}{2} \rho v^{2}+\frac{B^{2}}{8 \pi},
\end{aligned}
$$

where $\mathbf{v}, \mathbf{B}, E_{\text {total }}, \kappa_{\mathrm{r}}$, and $\Gamma$ are the fluid velocity, the magnetic field, the total energy, the radiative diffusivity, and the time independent cooling around the top boundary, respectively. We adopt the ideal equation of state $p=R \rho T$, where $R$ is the gas constant and the ratio of the heat constant is $\gamma=c_{\mathrm{p}} / c_{\mathrm{v}}=5 / 3$, where $c_{\mathrm{v}}$ is the heat capacity at constant volume. We use forth-order centered differences to discretize the problem in space, and the four-step Runge-Kutta method for time the integration (Vögler et al. 2005). The artificial viscosity suggested by Rempel et al. (2009) and Rempel (2014) is used to maintain stability. The artificial viscosity adopts slope-limiter which defines the slope of variables in a grid. Thanks to the slope limiter, we are able to minimize the diffusion in a smooth profile and maximize it at a steep gradient.

For initial conditions, we take a hydrostatic equilibrium with $d p / d x=-\rho g$. The 
temperature gradient is expressed as $\nabla=d \ln T / d \ln p$. Thus the initial conditions are

$$
\begin{aligned}
T_{0} & =T_{\mathrm{b}}\left[1-\nabla \frac{x}{H_{\mathrm{b}}}\right], \\
p_{0} & =p_{\mathrm{b}}\left[1-\nabla \frac{x}{H_{\mathrm{b}}}\right]^{1 / \nabla}, \\
\rho_{0} & =\rho_{\mathrm{b}}\left[1-\nabla \frac{x}{H_{\mathrm{b}}}\right]^{1 / \nabla-1},
\end{aligned}
$$

where $T_{\mathrm{b}}, p_{\mathrm{b}}, \rho_{\mathrm{b}}$, and $H_{\mathrm{b}}=-\left.(d \ln p / d x)^{-1}\right|_{x=0}=p_{\mathrm{b}} / \rho_{\mathrm{b}} g$ are the temperature, the pressure, the density, and the pressure scale height at $x=0$, which is the base of the CZ. The calculation domain is $(-1,0,0)<(x, y, z) / H_{\mathrm{b}}<(1.8,5.6,5.6)$ for cases with the RZ and $(0,0,0)<(x, y, z) / H_{\mathrm{b}}<(1.8,5.6,5.6)$ for cases without the RZ. Although calculations in this study are non-dimensional, the horizontal box size corresponds to $340 \mathrm{Mm} \sim 0.5 R_{\odot}$ when we fix the spatial scales by reference to $H_{\mathrm{b}}=60 \mathrm{Mm}$.

We use a stable stratification below $x=0$ (Layer I) and initially neutral above $x=0$ (Layer II). In the Layer I, the superadiabaticity $\delta$ is set to -0.2 , where the adiabatic temperature gradient is adopted in the Layer I (in the initial condition). We adopt an impenetratable boundary condition for the velocity, i.e. $v_{x}=\partial v_{y} / \partial x=\partial v_{z} / \partial x=0$ at both the top and bottom boundaries. At the top boundary only the vertical magnetic field is allowed, $\partial B_{x} / \partial x=B_{y}=B_{z}=0$. Only horizontal magnetic field is allowed at the bottom boundary, $B_{x}=\partial B_{y} / \partial x=\partial B_{z} / \partial x=0$. Periodic boundary condition is adopted for the all variables in the horizontal directions.

After calculations start, the Layer II becomes superadiabatic. $\Gamma$ is the time independent cooling around the top boundary expressed as:

$$
\begin{aligned}
F & =F_{0} \exp \left[-\left(\frac{x-x_{\max }}{d_{\max }}\right)^{2}\right], \\
\Gamma & =-\frac{d F}{d x}
\end{aligned}
$$

where $x_{\max }=1.8 H_{\mathrm{b}}$ is the location of top boundary and we set $d_{\max }=0.2 H_{\mathrm{b}} . F_{0}$ is the 
imposed flux at the base by the radiative diffusion.

Since all the energy flux is transported by the radiation in the $\mathrm{RZ}$, the radiative diffusion coefficient is defined only by the imposed energy flux and the temperature gradient as:

$$
\kappa_{\mathrm{r}}=-\frac{F_{0}}{d T_{0} / d x} \quad(x<0)
$$

Around the base of the $\mathrm{CZ}$, some fraction of the energy is transported by the radiation in the Sun. Thus we mimic this situation with decreasing the radiative diffusivity as:

$$
\kappa_{\mathrm{r}}=-\frac{F_{0}}{d T_{0} / d x} \exp \left[-\left(\frac{x}{d_{\text {min }}}\right)^{2}\right] \quad(x \geq 0),
$$

where $d_{\min }=0.4 H_{\mathrm{b}}$. Fig. 1 shows the radiative flux $\left(-\kappa_{\mathrm{r}} d T_{0} / d x\right.$ : black $)$ and the cooling around the top boundary (F: blue).

Although we start calculations with the simple analytic solution of hydrostatic balance, this initial condition changes in the RZ in order to reach an energy balance in the overshoot layer. We define normalized energy flux as $\bar{F}_{0}=F_{0} / \rho_{\mathrm{b}} c_{\mathrm{b}}^{3}$, where $\rho_{\mathrm{b}}$ and $c_{\mathrm{b}}^{3}$ are the density and the speed of sound at the base of CZ.

When the normalized energy flux is small $\left(\bar{F}_{0}<<1\right)$, this change takes a long time compared to thermal convection. In order to shorten this process, to keep the calculation reasonable, we adopt a similar method to Miesch et al. (2000) and Brun et al. (2011). In these papers, they increased the radiative diffusion in the $\mathrm{RZ}(x<0)$ in response to the enthalpy flux. In this paper, we increase the radiative diffusion $\kappa_{\mathrm{r}}$ to:

$$
\kappa_{\mathrm{r}}=\frac{F_{0}-F_{\mathrm{s}}}{d \bar{T} / d t}
$$

where the total $\left(F_{\mathrm{s}}\right)$, the enthalpy $\left(F_{\mathrm{e}}\right)$, the kinetic $\left(F_{\mathrm{k}}\right)$, and Poynting $\left(F_{\mathrm{m}}\right)$ fluxes are 


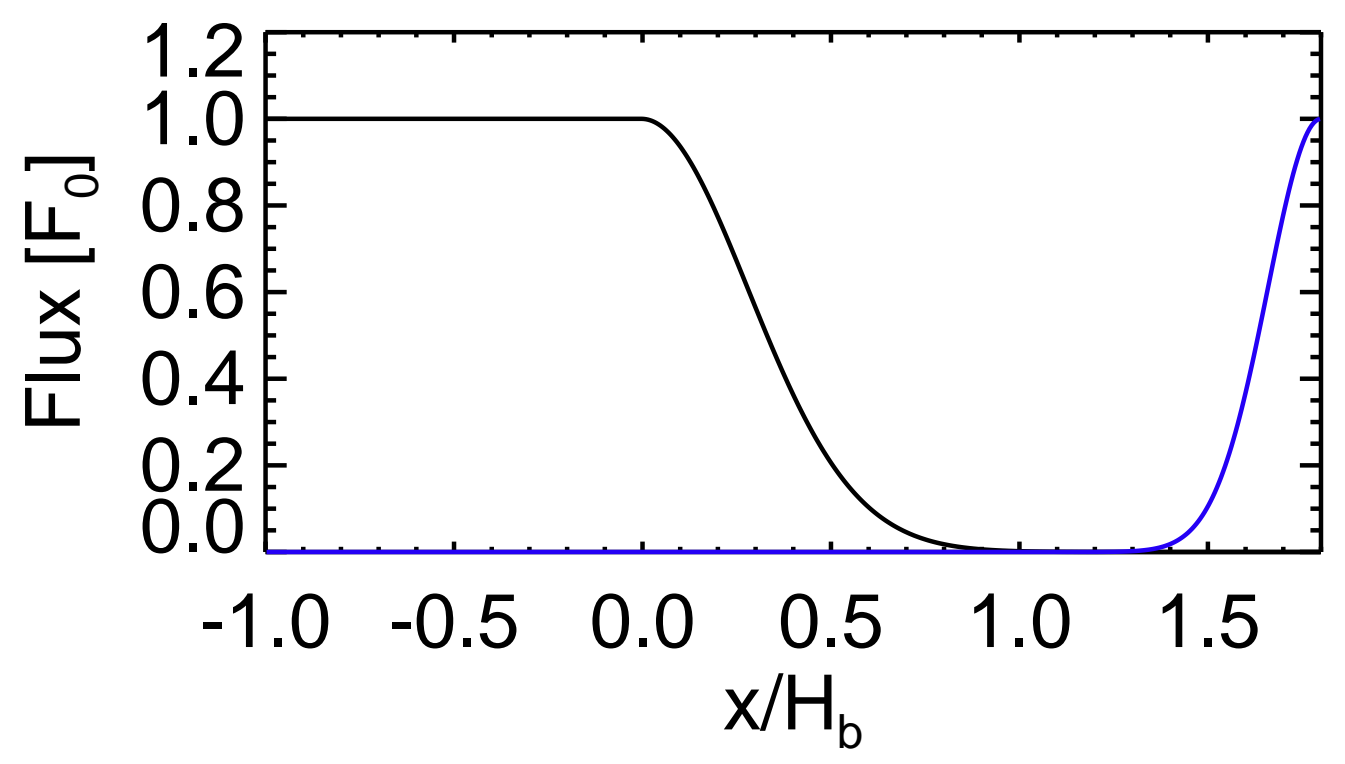

Fig. 1.- The radiative flux $\left(-\kappa_{\mathrm{r}} d T_{0} / d x\right.$ : black $)$ and the cooling around the top boundary (F: blue) are shown. The fluxes are normalized with the imposed flux $F_{0}$. 
defined as:

$$
\begin{aligned}
F_{\mathrm{s}} & =F_{\mathrm{e}}+F_{\mathrm{k}}+F_{\mathrm{m}}+F_{\mathrm{a}}, \\
F_{\mathrm{e}} & =\int c_{\mathrm{p}} T\left(\rho v_{x}-\overline{\rho v_{x}}\right) d S / \int d S, \\
F_{\mathrm{k}} & =\int \frac{1}{2} v^{2}\left(\rho v_{x}-\overline{\rho v_{x}}\right) d S / \int d S, \\
F_{\mathrm{m}} & =\int \frac{1}{4 \pi}\left(B^{2} v_{x}-\mathbf{v} \cdot \mathbf{B} B_{x}\right) d S / \int d S,
\end{aligned}
$$

where overlines on the variables expresses horizontal averages. Here $F_{\mathrm{a}}$ is the energy flux due to the artificial viscosity on the internal energy (see the detail in Rempel 2014). Here, we investigate the downflow penetration and dynamo in this state (further discussion is seen around Fig. (7).

In order to compare results from simulations with significantly different energy fluxes, we define "convection velocity $\left(v_{\mathrm{c}}\right)$ ". As discussed in the introduction, the normalized energy flux determines the superadiabaticity in the $\mathrm{CZ}$ as $\delta \sim \bar{F}_{0}^{2 / 3}$. We change the normalized energy flux and study the dependence of the convection and dynamo behavior. From the relation of the energy flux and the convection velocity $F_{0} \sim \rho_{0} v_{\mathrm{c}}^{3}$, the convection velocity is expressed as:

$$
v_{\mathrm{c}}=\bar{F}_{0}^{1 / 3} c_{\mathrm{b}}
$$

We use this value $\left(v_{\mathrm{c}}\right)$ for the normalization.

We study 24 cases in total. All the cases are summarized in Tables 11. For the cases with a RZ, we choose three different normalized energy fluxes, $\bar{F}_{0}=5 \times 10^{-5}, 5 \times 10^{-6}$, and $5 \times 10^{-7}$. In the highest resolution cases, the grid spacing is $\Delta x=\Delta y=\Delta z \sim 5.5 \times 10^{-3} H_{\mathrm{b}}$. This corresponds to $330 \mathrm{~km}$ on the Sun. Thermal convection in the $\mathrm{CZ}$ is largely uninfluenced by imposed energy flux, when we consider quantities normalized with the convection velocity $v_{\mathrm{c}}=\bar{F}_{0}^{1 / 3} c_{\mathrm{b}}$. Thus we do not change the normalized energy flux 
$\left(\bar{F}_{0}=5 \times 10^{-6}\right)$ for the cases without the RZ. We carry out the calculations with three different resolutions, High, Medium, and Low. The numbers of the grid points are shown in Tables 1. In order to use identical grid spacings for the cases with and without RZ, the number of grid points in the $x$ direction is changed, correspondingly. We mainly analyze the case high resolution series. For all the cases, we carry out calculations with and without the magnetic field. For the cases with the magnetic field, we initially inject a weak seed magnetic field. All graphics and analysis presented in this study correspond to the High series (unless otherwise stated)

\section{Result}

We now show the overall convection patterns of the calculations. Fig. 2 shows vertical cuts of the vertical velocity $\left(v_{x}\right)$. The results with the normalized energy flux of (a) $\bar{F}_{0}=5 \times 10^{-5}$ without the magnetic field, (b) $\bar{F}_{0}=5 \times 10^{-5}$ with the magnetic field, and (c) $\bar{F}_{0}=5 \times 10^{-7}$ with the magnetic field are shown. Perturbations in the RZ $(x<0)$ correspond to internal gravity waves. Since the temperature gradient is almost the same in all cases in the RZ, the radiative diffusion $\left(\kappa_{\mathrm{r}}\right)$ is proportional to the energy flux imposed at the bottom boundary. Thus the typical wavelength is short with the small energy flux

Table 1: Free parameters for calculations. As a total, $3 \times 4 \times 2=24$ cases are calculated. The numbers in the square brackets show the number of grid points in $\mathrm{x}$ direction for the cases without the RZ.

\begin{tabular}{l|c}
\hline \hline Grid points & \\
$\left(N_{x}, N_{y}, N_{z}\right)$ & $(128[88], 256,256),(256[176], 512,512),(512[376], 1024,1024)$ \\
Normalized flux $\bar{F}_{0}$ & $5 \times 10^{-5}, 5 \times 10^{-6}, 5 \times 10^{-7}(\mathrm{w} / \mathrm{RZ}), 5 \times 10^{-6}(\mathrm{w} / \mathrm{o} \mathrm{RZ})$ \\
Magnetic field & Yes, No \\
\hline \hline
\end{tabular}



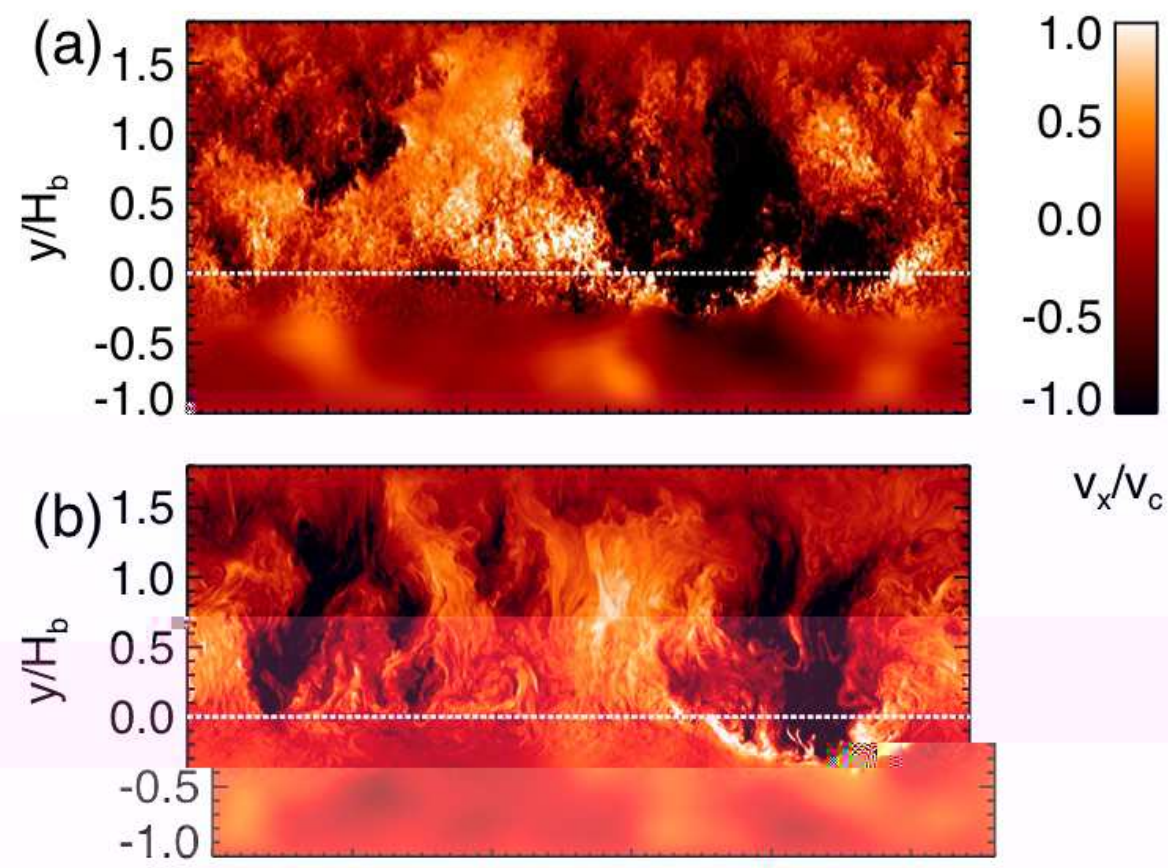

$\mathrm{v}_{\mathrm{x}} / \mathrm{v}_{\mathrm{c}}$

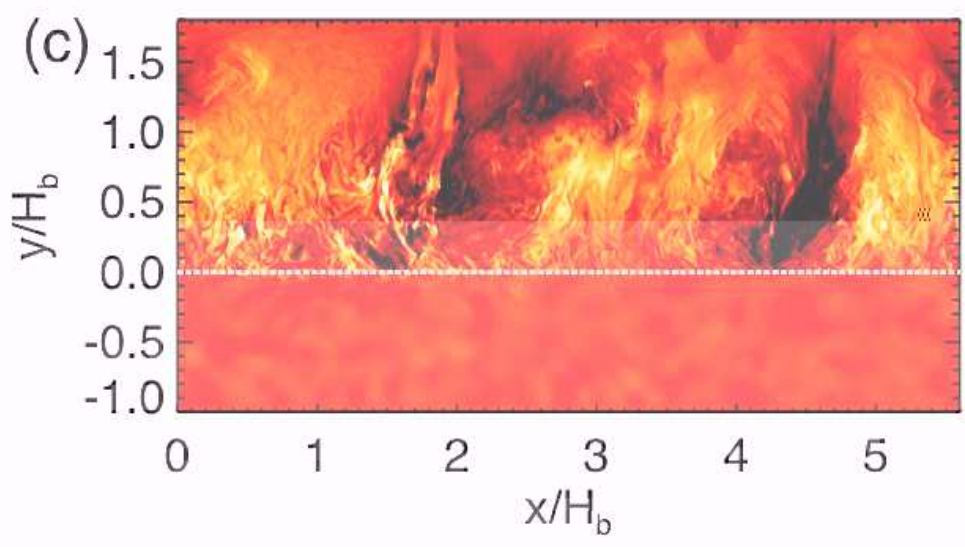

Fig. 2.- Vertical cross sections of the vertical velocity $\left(v_{x}\right)$. The results with $(\mathrm{a}) \bar{F}_{0}=$ $5 \times 10^{-5}$ without the magnetic field, (b) $\bar{F}_{0}=5 \times 10^{-5}$ with the magnetic field, and (c) $\bar{F}_{0}=5 \times 10^{-7}$ with the magnetic field are shown. White dashed lines shows $x=0$, where the location of the transition between the $\mathrm{CZ}$ and the RZ. 

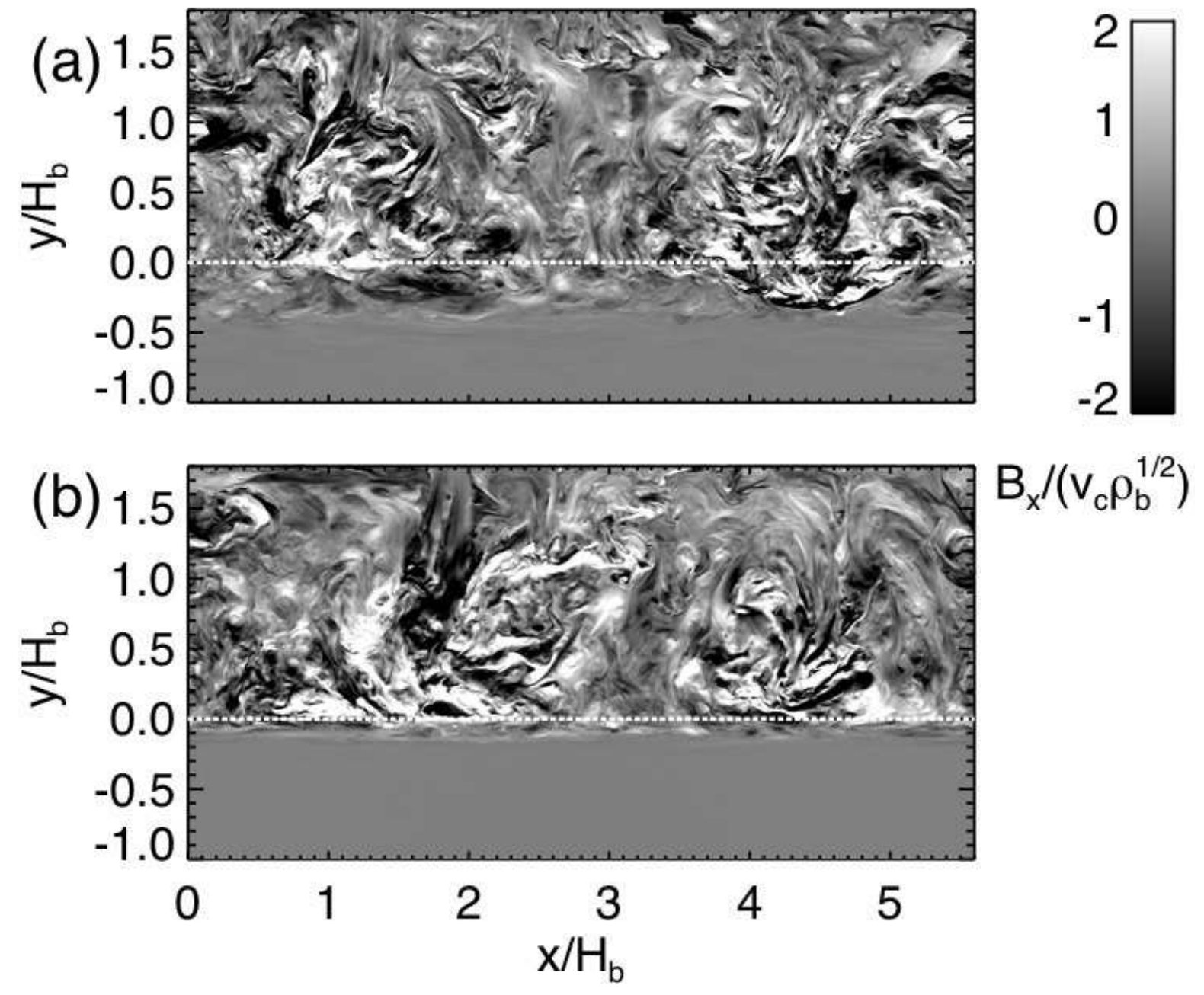

Fig. 3.- Vertical cross sections of the vertical magnetic field $\left(B_{x}\right)$. The results with (a) $\bar{F}_{0}=5 \times 10^{-5}$ and (b) $5 \times 10^{-7}$ are shown. White dashed lines show $x=0$, where the location of the transition between the $\mathrm{CZ}$ and the RZ. 


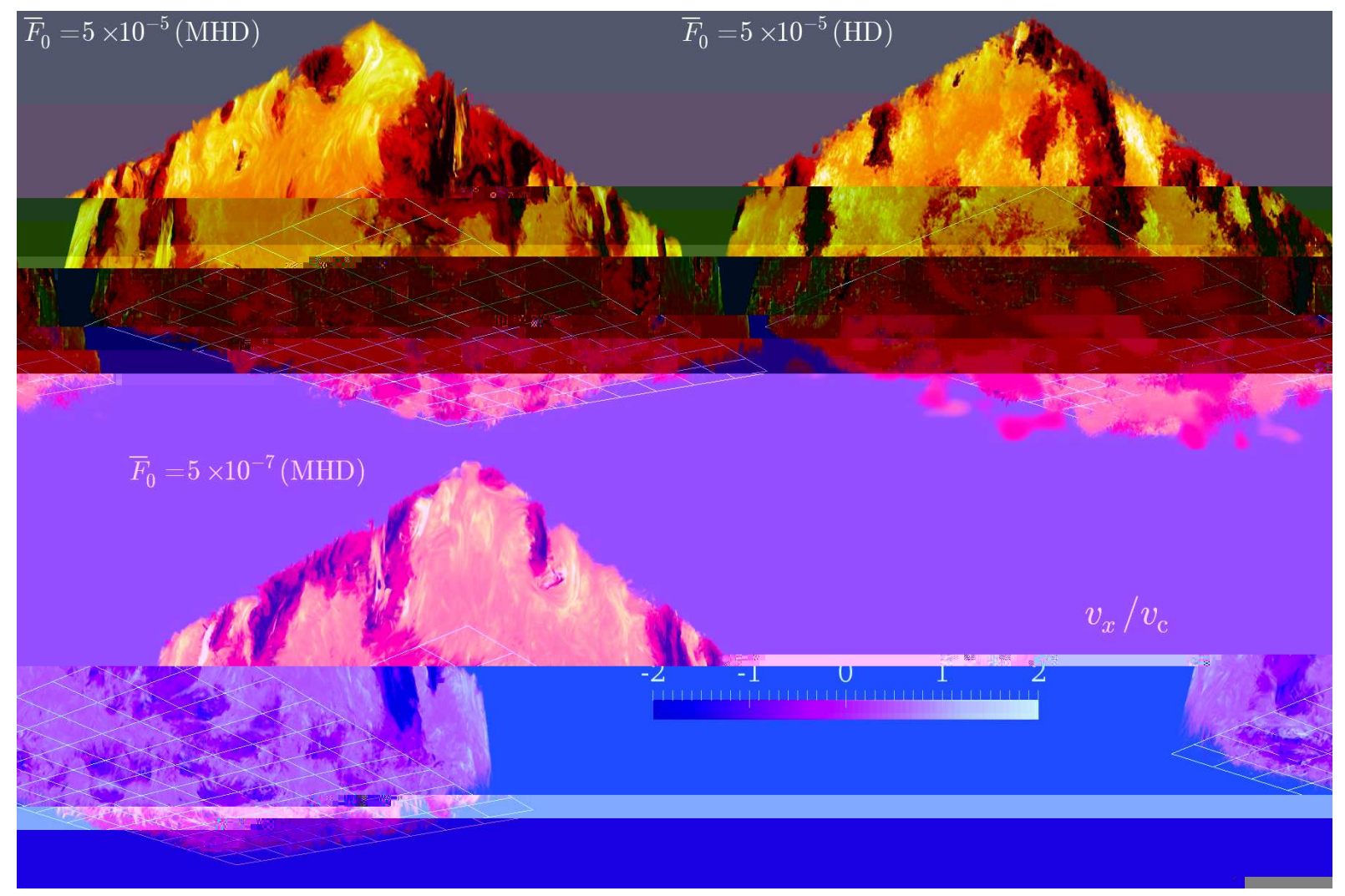

Fig. 4. $-3 \mathrm{D}$ visualization of the calculations. The results with $\bar{F}_{0}=5 \times 10^{-5}$ with the magnetic field (top-left), $\bar{F}_{0}=5 \times 10^{-5}$ without the magnetic field (top-right), and $\bar{F}_{0}=5 \times 10^{-7}$ with the magnetic field (bottom-left) are shown. Volume rendered vertical velocity $\left(v_{x}\right)$ is shown. The white grid lines at $x=0$ show the base of the CZ. Animation is also available online. 
(panel c).

As shown in Hotta et al. (2015a), an efficient small-scale dynamo generates strong small-scale magnetic field which significantly suppresses the small-scale velocity features (Panels a and b). A comparison between panels b $\left(\bar{F}_{0}=5 \times 10^{-5}\right)$ and $\mathrm{c}\left(\bar{F}_{0}=5 \times 10^{-7}\right)$ shows that the overall features of the CZ are similar, but penetration in the OS is significantly different. Similar features are seen in figures of the magnetic field. Figure 3 shows the vertical magnetic field $\left(B_{x}\right)$ on a vertical cut. Panels a and b show the results with $\bar{F}_{0}=5 \times 10^{-5}$ and $5 \times 10^{-7}$, respectively. Small energy fluxes results in shallow penetration and overall feature in the $\mathrm{CZ}(x>0)$ are similar with different energy fluxes. Three-dimensional visualization nicely shows features of the calculations in Fig. 4. An animation of the figure is also available online.

(a)

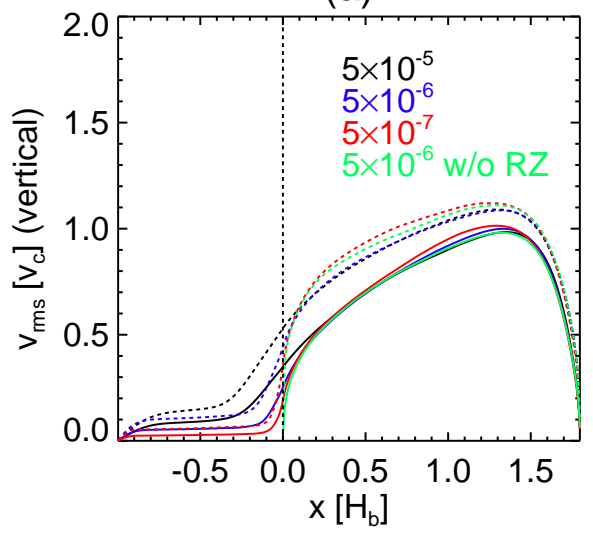

(b)

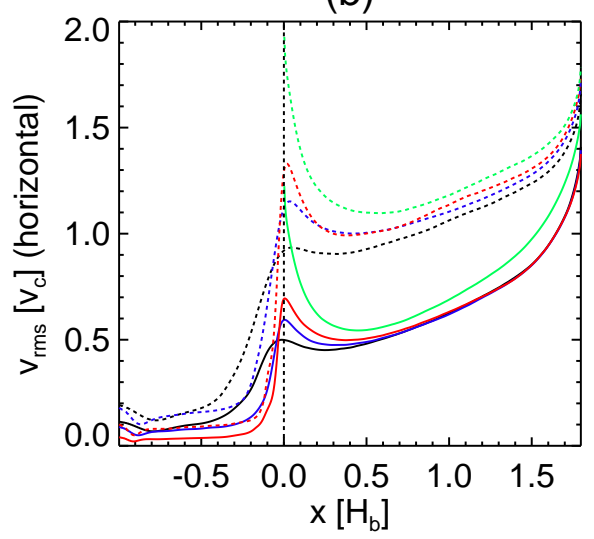

Fig. 5.- Vertical $\left(v_{x}\right.$ : panel a) and horizontal $\left(\sqrt{v_{y}^{2}+v_{z}^{2}}\right.$ : panel b) normalized RMS velocities. Solid and dashed lines show the calculations with and without the magnetic field, respectively. The results with the normalized energy flux of $\bar{F}_{0}=5 \times 10^{-5}$ (black), $5 \times 10^{-6}$ (blue), and $5 \times 10^{-7}$ (red) are shown. The green lines show the results without the RZ and with $\bar{F}_{0}=5 \times 10^{-6}$.

Figure 5 shows the vertical (panel a) and horizontal (panel b) normalized root-mean- 
square (RMS) velocities with different energy fluxes $\left(\bar{F}_{0}\right)$. The RMS velocity is defined as $v_{\text {rms }}=\sqrt{\left\langle(v-\langle v\rangle)^{2}\right\rangle}$, where the bracket indicates the horizontal average. The result with $\bar{F}_{0}=5 \times 10^{-5}$ (black), $5 \times 10^{-6}$ (blue), and $5 \times 10^{-7}$ (red) are shown. Solid and dashed lines show the results with and without the magnetic field, respectively. Green lines show the results without the RZ. As shown in Hotta et al. (2015a), the normalized RMS velocity is significantly suppressed with the magnetic field, due to the high resolution (low diffusivities) and efficient small-scale dynamo. As expected, Fig. 2 with a smaller energy flux has a shallower penetration depth. The details of the penetration depth is discussed later. The result without the RZ has the largest horizontal normalized RMS velocity, since there is artificial boundary at the bottom. In the cases with the RZ, the smaller energy flux increases the normalized RMS velocity. This result indicates that the RZ becomes relatively stiffer with the smaller energy flux. The circulation of the $\mathrm{CZ}$ is then promoted and a sudden suppression of the downflow at the base of the CZ is observed. This generates a sudden increase of the horizontal flow at the base of the CZ (panel b). Interestingly, the results with the magnetic field are almost the same in cases with different energy flux (solid line). This is caused by the stronger magnetic field with smaller energy flux. This is also discussed later.

Figure 6] shows the dependence of the overshoot depth $\left(d_{\mathrm{os}}\right)$, i.e., the penetration depth dependence on the energy flux and the resolution. The overshoot depth is defined with the normalized RMS velocity. We define that the base of the overshoot region is the place where the normalized vertical RMS velocity is $1 / e$ of that at the base of $\mathrm{CZ} x=0$. Panel a shows the results from the cases Low (black), Medium (blue), and High (red) with the magnetic field. Smaller energy fluxes make the overshoot depth small. The cases with different resolutions clearly shows that a higher resolution results in a shallower penetration. When we increase the number of grid points, the effect of the artificial viscosity can be decreased. This makes the overshoot region thin. The resolution dependence becomes most prominent 
(a)

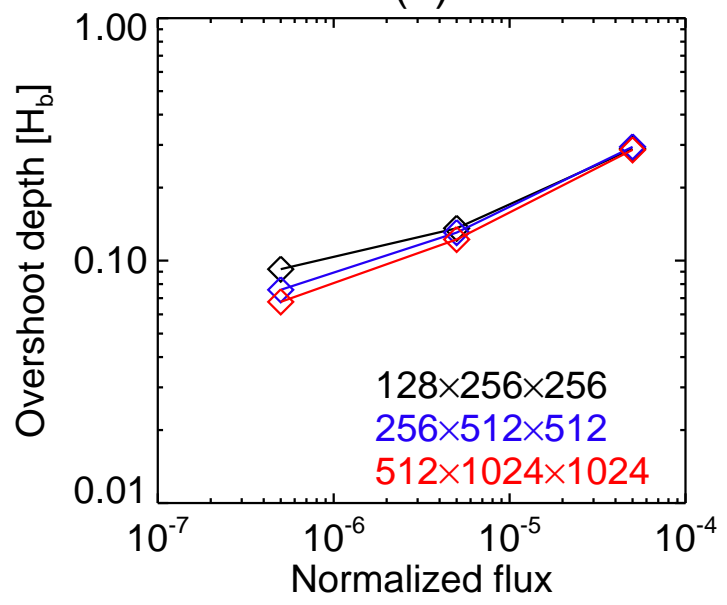

(b)

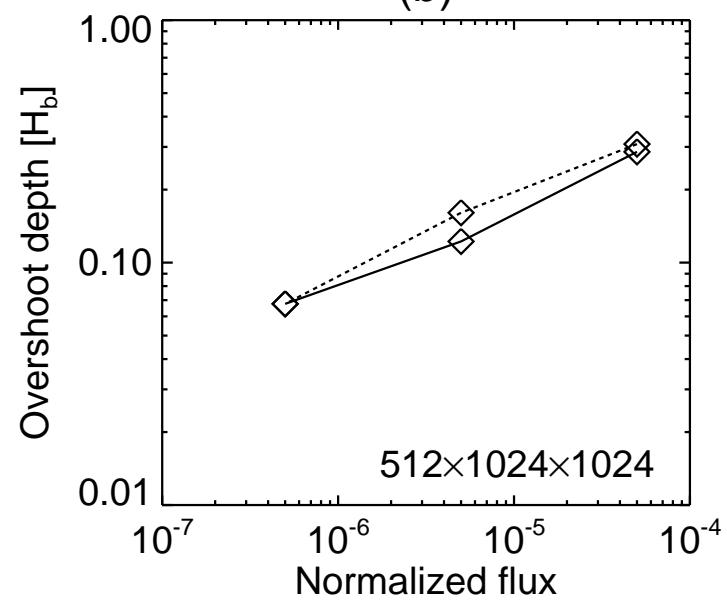

Fig. 6.- Overshoot depth with different settings is shown. Panel a shows dependence of overshoot depth on the energy flux and the resolution. The result from the cases Low (black), Medium (blue), and High (red) series with the magnetic field are shown. Panel b are from the cases High series. Dashed and solid lines show the results without and with the magnetic field, respectively. 
when the energy flux is small $\left(\bar{F}_{0}=5 \times 10^{-7}\right)$, since the overshoot depth is small and a large number of grid points is required. With the least-square fit with the results of the cases high resolution series leads reveals a relation between the overshoot depth and the energy flux as $d_{\mathrm{os}} / H_{\mathrm{b}} \sim 6.1 \bar{F}_{0}^{0.31}$. We can estimate the solar overshoot depth $d_{\mathrm{os}} \sim 0.004 H_{\mathrm{b}} \sim 250 \mathrm{~km}$ when we extrapolate to $\overline{F_{0}} \sim 5 \times 10^{-11}$. Panel b shows a comparison of the results with and without the magnetic field. The dashed and solid lines shows the results without and with the magnetic field, respectively. The cases with the magnetic field tend to show shallower penetrations. The magnetic field suppresses the convection velocity, significantly in the CZ. Since the superadiabaticity in the RZ is kept, the RZ becomes relatively stiff with the magnetic field.

(a)

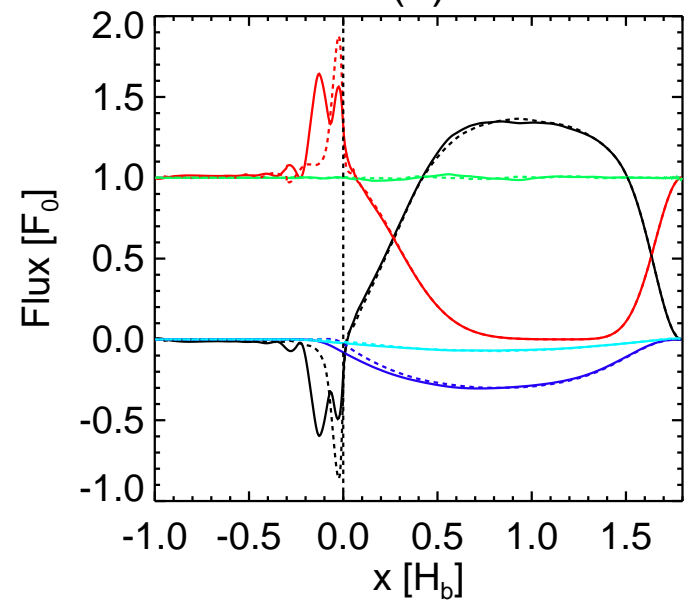

(b)

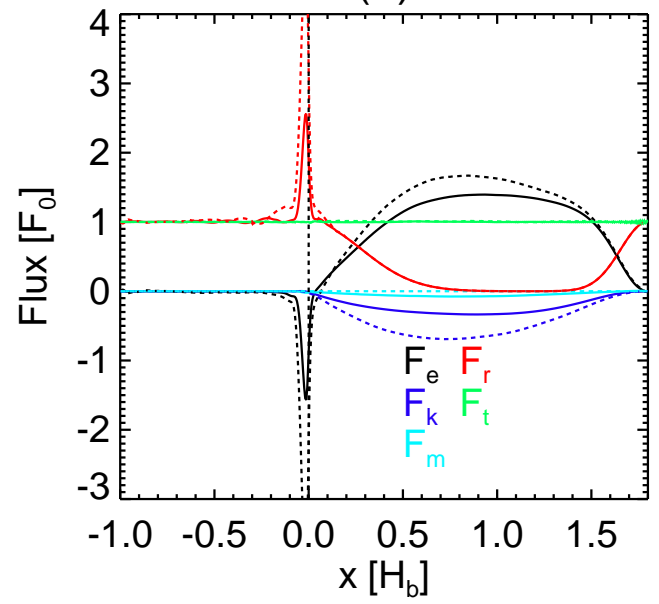

Fig. 7.- The enthalpy $\left(F_{\mathrm{e}}\right.$ : black $)$, the radiative $\left(F_{\mathrm{r}}\right.$ : red $)$, the kinetic $\left(F_{\mathrm{k}}\right.$ : blue), the Poynting $\left(F_{\mathrm{m}}\right.$ : light blue) and the total $\left(F_{\mathrm{t}}\right.$ : green $)$ energy flux are shown. Here the radiative flux includes the artificial cooling in order to avoid complexity in the figure. (a) Solid and dashed lines shows the results with $\bar{F}_{0}=5 \times 10^{-5}$ and $5 \times 10^{-6}$, respectively. Both results have the magnetic field. (b) Solid and dashed lines show the result with and without the magnetic field, respectively. The energy flux in both cases is $\bar{F}_{0}=5 \times 10^{-7}$. 
Figure 7 shows the enthalpy $\left(F_{\mathrm{e}}\right.$ : black $)$, the radiative $\left(F_{\mathrm{r}}\right.$ : red $)$, the kinetic $\left(F_{\mathrm{k}}\right.$ : blue), the Poynting $\left(F_{\mathrm{m}}\right.$ : light blue) and the total $\left(F_{\mathrm{t}}\right.$ : green) energy flux. In order to avoid complexity in the figure, the radiative flux at the bottom and cooling flux at top boundary (F: eq. 11) are united to the radiative flux $\left(F_{\mathrm{r}}=-\kappa_{\mathrm{r}} d T / d r+F\right)$. Panel a shows the results with $\bar{F}_{0}=5 \times 10^{-5}$ (solid) and $5 \times 10^{-6}$ (dashed). Both cases include the magnetic field. In spite of the difference of the energy flux, the overall feature in the $\mathrm{CZ}$ is not very much different. The enthalpy flux in the OS $(x<0)$ is much more different in the different cases. The smaller energy fluxes shows a steep and large negative peak of the enthalpy flux in the OS. Panel b shows the result with $\bar{F}_{0}=5 \times 10^{-7}$ with (solid) and without (dashed) the magnetic field. The peak of the enthalpy flux is decreased significantly with the magnetic field in the OS, since the convection velocity is decreased.

Fig. 8 shows the superadiabaticity. The results with $\bar{F}_{0}=5 \times 10^{-5}$ (black), $5 \times 10^{-6}$ (blue), and $5 \times 10^{-7}$ (red) with the magnetic field are shown. As explained in the introduction, the superadiabaticity in the CZ is smaller with smaller energy flux. An interesting finding is the change of the sign in the superadiabaticity occurs above the OS (around $x=0.5 H_{\mathrm{b}}$ ). This means that a subadiabatic (convectively stable) region exists in the CZ. When there is not the subadiabatic layer around the base of the CZ, the low entropy downflow directly breaks into the RZ. When the RZ is stiff, most of low entropy fluid is reflected and the low entropy accumulates around the base of the CZ. This causes the positive entropy gradient, i.e., the subadiabatic region around the base of the CZ. This should exist even in the Sun, because the solar RZ is much stiffer than what we can achieve in this paper. The subadiabatic CZ is also found in non-local mixing model (Rempel 2004). Once the subadiabatic region is constructed, the downflow is decelerated by the buoyancy even in the $\mathrm{CZ}$.

Figure 9 shows the normalized RMS magnetic field with different energy fluxes. The 


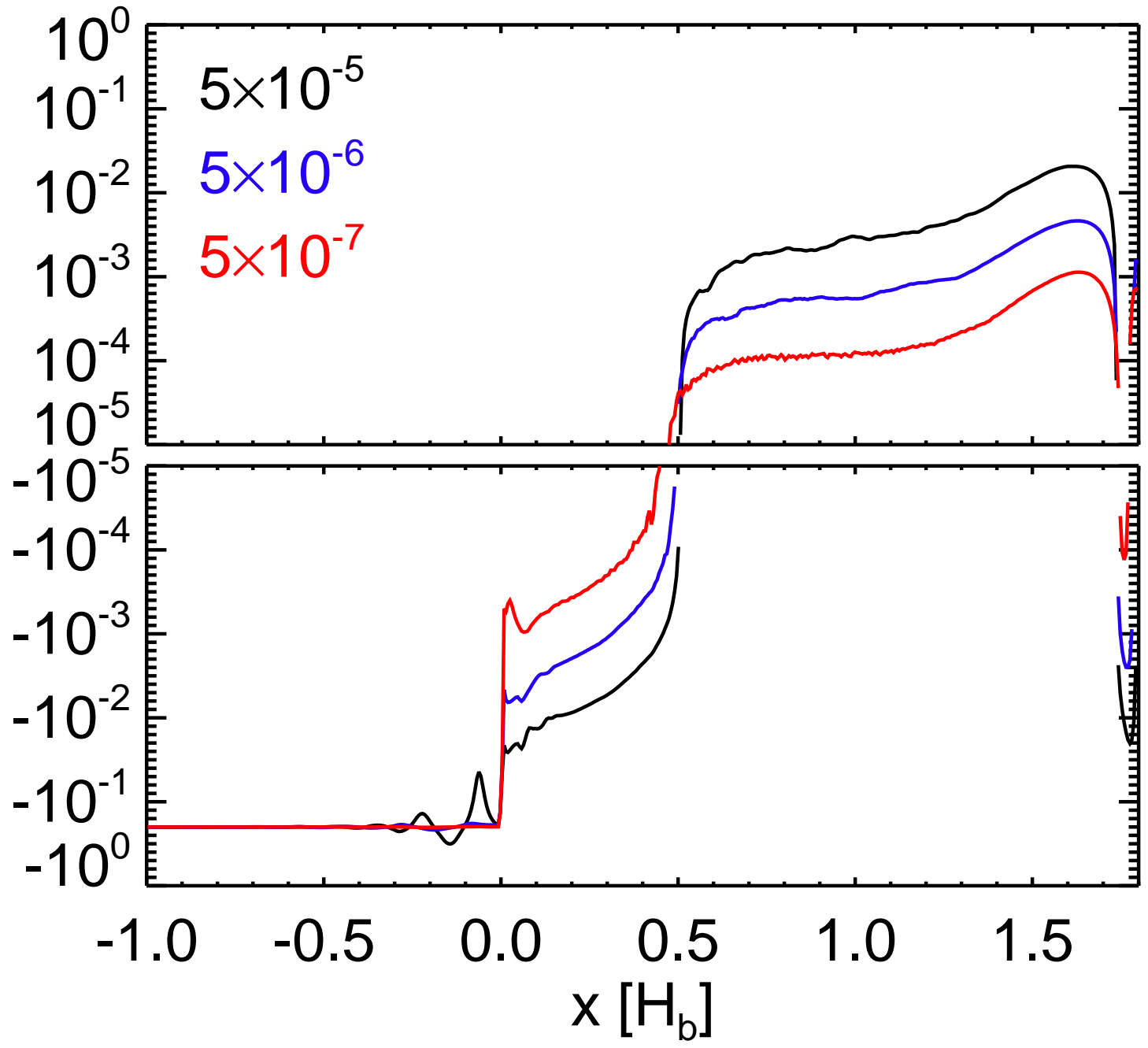

Fig. 8.- Superadiabaticity $\delta$ with different setting is shown. The results with $\bar{F}_{0}=5 \times 10^{-5}$ (black), $5 \times 10^{-6}$ (blue), and $5 \times 10^{-7}$ (red) with the magnetic field are shown. 


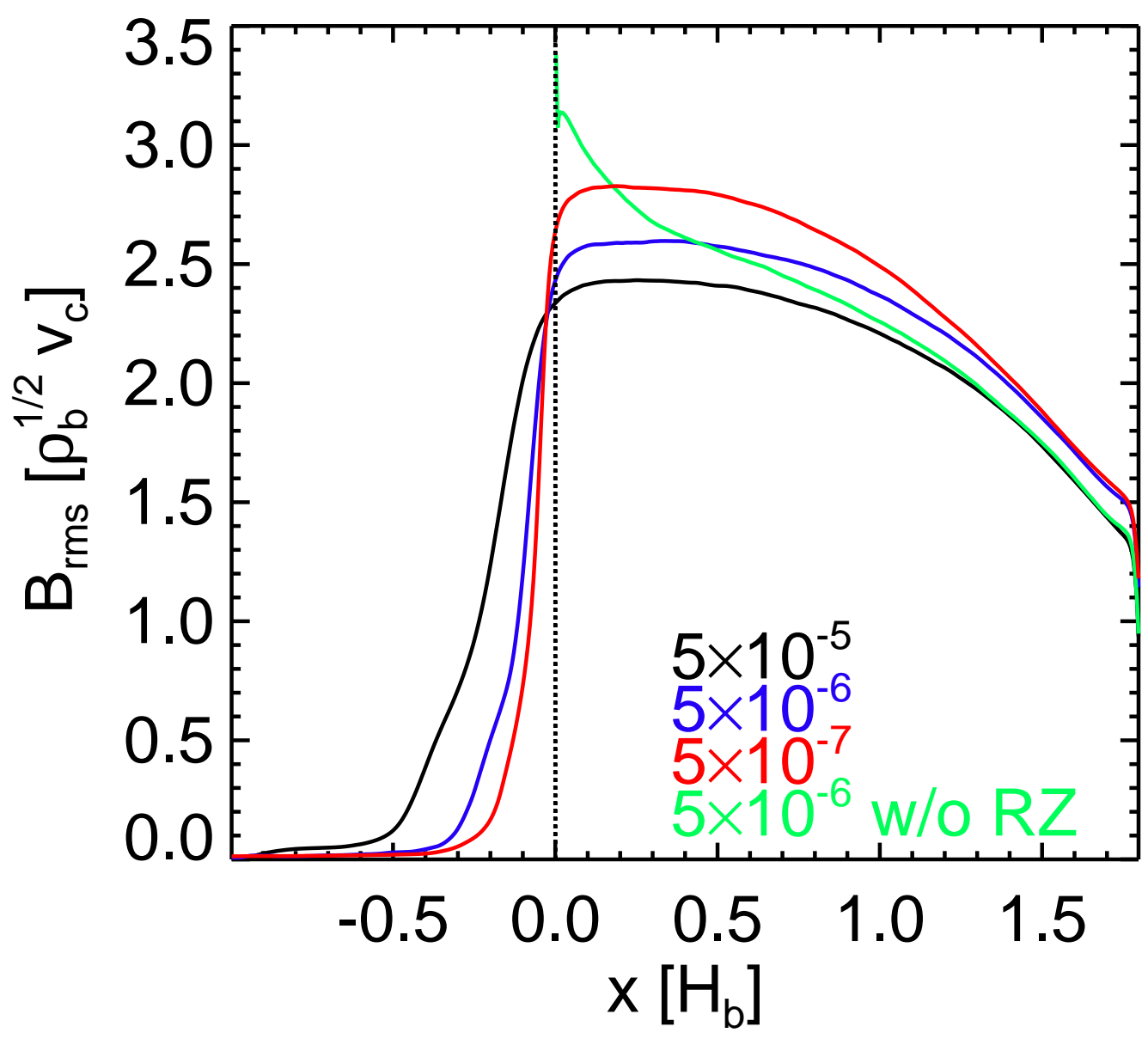

Fig. 9.- Normalized RMS mangetic field is shown. The results with $\bar{F}_{0}=5 \times 10^{-5}$ (black), $5 \times 10^{-6}$ (blue), and $5 \times 10^{-7}$ (red) with the RZ are shown. The green line shows the result without the RZ. 
results with $\bar{F}_{0}=5 \times 10^{-5}$ (black), $5 \times 10^{-6}$ (blue), and $5 \times 10^{-7}$ (red) with the RZ are shown. The green line shows the result without the RZ. As shown in Fig. 3, shallower penetration with smaller energy flux is seen. In addition, the normalized RMS magnetic field in the $\mathrm{CZ}$ is increased with a decrease of the energy flux. The case without the RZ is an extreme of the small energy flux, i.e., stiff RZ. While the artificial wall increases the magnetic field strength around the base of the $\mathrm{CZ}$, the magnetic field in the $\mathrm{CZ}$ (green) is weaker than in those cases with a RZ. This could be caused by efficient accumulation of the magnetic field at the base of the CZ. The result indicates that the efficiently accumulated magnetic energy at base of the $\mathrm{CZ}$ with the artificial boundary is less transported to the upper part of the CZ than that with the RZ. Since we cannot achieve the real stiffness of the solar RZ in this study, the realistic situation should be somewhere between the results with smallest energy flux and without the RZ. Thanks to the high stiffness of the real sun, the overestimation introduced by the use of a wall boundary condition is not very severe.

Figure 10 shows the dependence of the magnetic field strength on the resolution and the energy flux. Panel a shows the normalized RMS magnetic field. The cases High (black), Medium (blue), and Low (red) series are shown. The increase of the resolution increases the magnetic field. This is a general tendency of the small-scale dynamo (Rempel 2014; Hotta et al. 2015a). The results also indicates that the smaller energy flux tends to produce stronger magnetic fields. The result with the smallest energy flux $\left(\bar{F}_{0}=5 \times 10^{-7}\right)$ in the cases low resolution series is not reliable, since the resolution is too low to resolve the thin OS and the contribution of the artificial viscosity is very large. Panel b shows the ratio of the normalized RMS magnetic field $\left(B_{\mathrm{rms}}\right)$ to the equipartition magnetic field $\left(B_{\mathrm{eq}}=\sqrt{4 \pi \rho_{0}} v_{\mathrm{rms}}\right)$. This value also increases with resolution. There is no clear dependence of this on the energy flux. In the following paragraph, we investigate the reason why the small energy flux has efficient small-scale dynamo. 
(a)

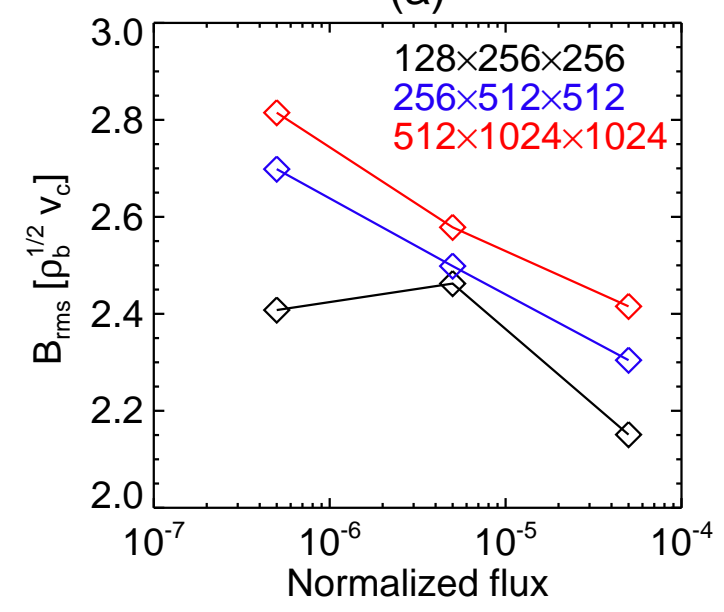

(b)

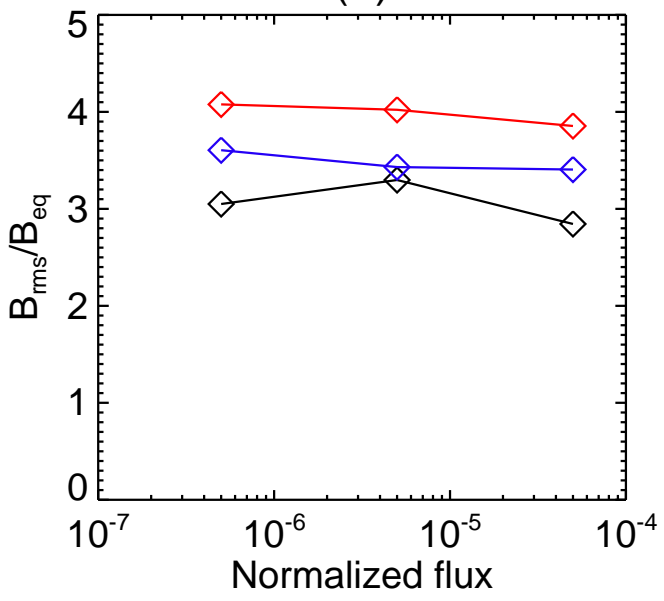

Fig. 10.- Dependence of the magnetic field strength on the resolution and the energy flux is shown. The results from the case High (black), Medium (blue), and Low series (red) are shown. Panel a and b show the normalized RMS magnetic field $\left(B_{\mathrm{rms}}\right)$ and the normalized RMS mangetic field divided by the equipartition magnetic field $\left(B_{\mathrm{rms}} / B_{\mathrm{eq}}\right)$ around the base of the $\mathrm{CZ}\left(x=0.1 H_{\mathrm{b}}\right)$, respectively. 
(a)

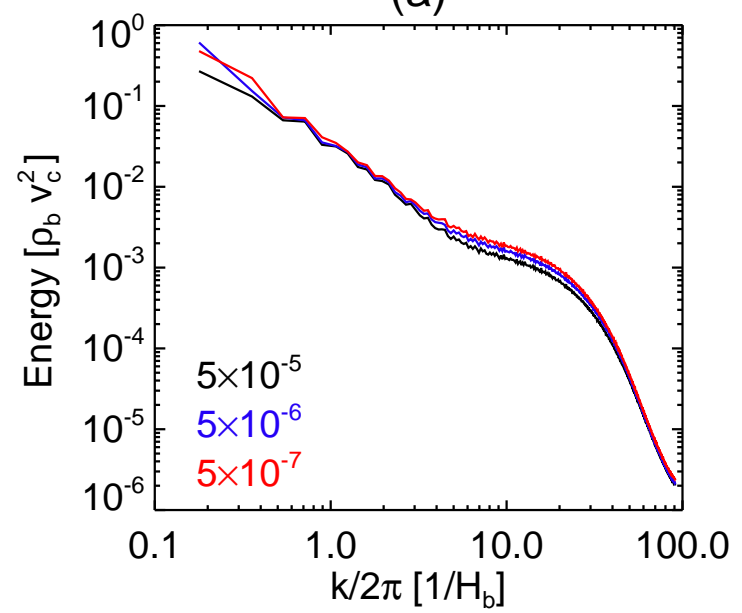

(b)

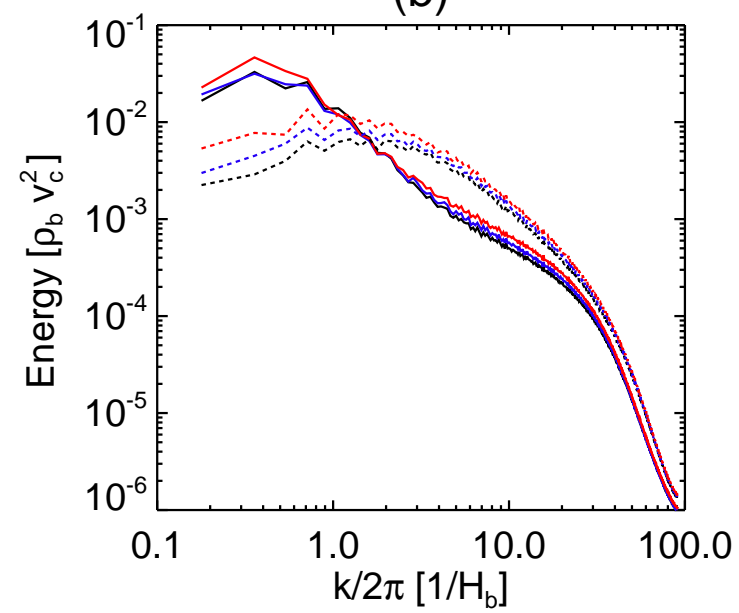

Fig. 11. - Energy spectra at the base of the CZ $\left(x=0.1 H_{\mathrm{b}}\right)$ are shown. Solid and dashed lines show the kinetic and magnetic energy, respectively. Panel a and b show the results without and with the magnetic field, respectively. The result with $\bar{F}_{0}=5 \times 10^{-5}$ (black), $5 \times 10^{-6}$ (blue), and $5 \times 10^{-7}(\mathrm{red})$ are shown.

Figure 11 shows the kinetic (solid) and magnetic (dashed) spectra at the base of the CZ $(x=0.1)$. Panel a and b show the results without and with the magnetic field, respectively (results with $\bar{F}_{0}=5 \times 10^{-5}$ (black), $5 \times 10^{-6}$ (blue), and $5 \times 10^{-7}$ (red) are shown). Small energy fluxes have large kinetic energies at small spatial scales $\left(k /(2 \pi)>1 / H_{\mathrm{b}}\right)$. This could be caused by the stiffer wall at the base of the $\mathrm{CZ}$. The stiff wall creates the small scale turbulence with the sudden suppression of the downflow. This creates the magnetic field through the small-scale dynamo.

Figure 12 shows a proxy of the inclination of the magnetic field $\left(B_{\mathrm{h}} / B\right)$, where $B_{\mathrm{h}}=\left(B_{y}^{2}+B_{z}^{2}\right)^{1 / 2}$. The mangnetic field becomes horizontal around the base of the $\mathrm{CZ}$ when the energy flux is small. This is caused by the stiff RZ. This is also discussed in the following paragraph. Interestingly the inclination in the $\mathrm{CZ}$ is not influenced by the energy flux. Although the calculation without the RZ slightly overestimates the inclination 


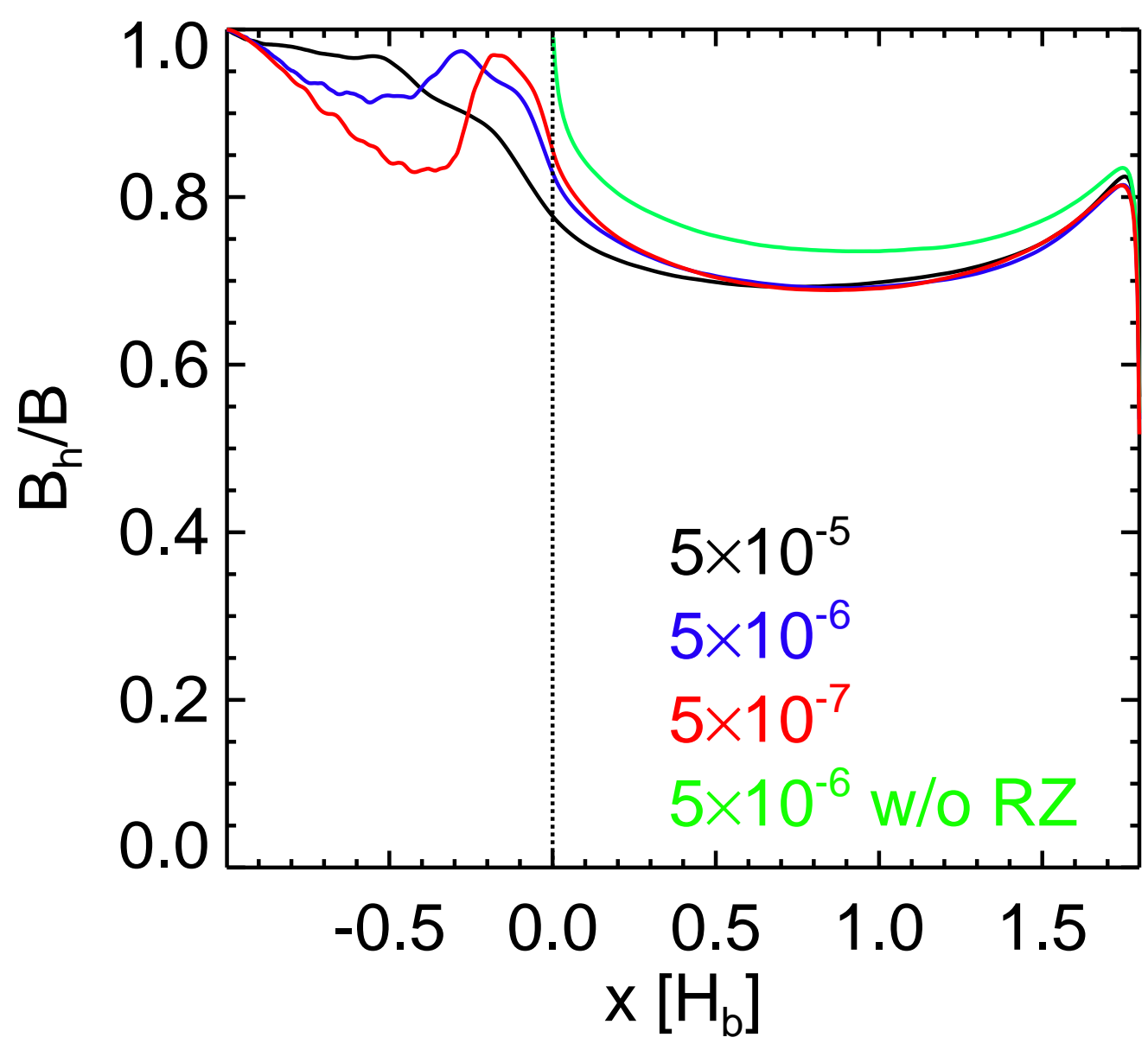

Fig. 12.- A proxy of the inclination of the magnetic field $\left(B_{\mathrm{h}} / B\right)$ is shown with different energy flux. The results with $\bar{F}_{0}=5 \times 10^{-5}$ (black), $5 \times 10^{-6}$ (blue), and $5 \times 10^{-7}$ (red)with the RZ are shown. The green line shows the result with $\bar{F}_{0}=5 \times 10^{-6}$ without the RZ. 
with the horizontal magnetic field boundary condition, the magnetic field is almost totally horizontal at the base of the $\mathrm{CZ}$ even with the RZ. We conclude that the horizontal magnetic field boundary condition at the base of the $\mathrm{CZ}$ is a good way to mimic the RZ, when we need to exclude the RZ.

In order to investigate the origin of the increase of the magnetic energy and the field inclination in the cases with the small energy flux, we analyze energy conversion terms in the equation of motion. The contributions to the work calculated to this purpose are listed:

$$
\begin{aligned}
W_{\text {buo }} & =-\rho g v_{x}-\frac{\partial p}{\partial x} v_{x}, \\
W_{\text {pre(h) }} & =-\frac{\partial p}{\partial y} v_{y}-\frac{\partial p}{\partial z} v_{z}, \\
W_{\text {lor }(\mathrm{v})} & =\frac{[(\nabla \times \mathbf{B}) \times \mathbf{B}]_{x}}{4 \pi} v_{x}, \\
W_{\text {lor }(\mathrm{h})} & =\frac{[(\nabla \times \mathbf{B}) \times \mathbf{B}]_{y}}{4 \pi} v_{y}+\frac{[(\nabla \times \mathbf{B}) \times \mathbf{B}]_{z}}{4 \pi} v_{z},
\end{aligned}
$$

where $W_{\text {buo }}, W_{\text {pre(h) }}, W_{\text {lor(v) }}$, and $W_{\text {lor(h) }}$ are the work done by the buoyancy, the horizontal pressure gradient, the vertical Lorentz force, and the horizontal Lorentz force, respectively. Fig. 13 shows horizontally averaged works with different energy fluxes. Panel a shows the buoyancy work $\left(W_{\text {buo }}\right)$. In the upper $\mathrm{CZ}$ which is superadiabatic, this work is positive. These works accelerate the thermal convection. Around the lower CZ, the work becomes negative due to the subadiabatic CZ. The contribution becomes larger with decreasing the energy flux. Most of deceleration is done in the $\mathrm{CZ}$ with $\bar{F}_{0}=5 \times 10^{-7}$, since the RZ is very stiff and the OS is very thin. Although the subadiabtic layer is seen blow $x=0.5 H_{\mathrm{b}}$ in Fig. 8. the deceleration by the buoyancy starts around $x=0.3 H_{\mathrm{b}}$. This is because the density change in the downflow does not occur immediately. When the downflow goes into the stable region from the upper layer, the downflow is heavier than the mean density. Thus the buoyancy work can be positive even in the subadiabtic region. After the downflow proceed certain distance, the density is modified and the buoyancy work becomes negative. Panel b 

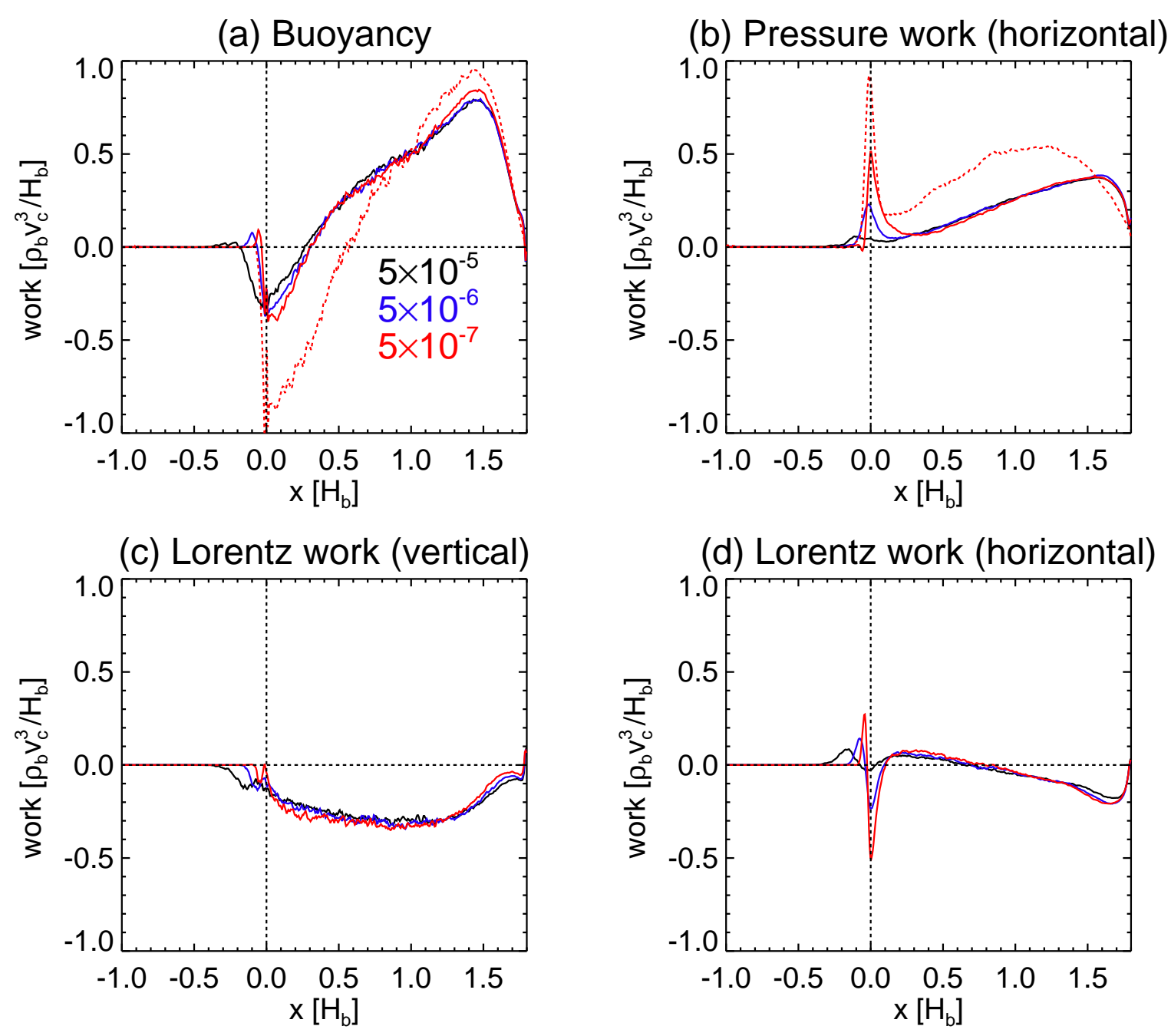

Fig. 13. - The works are shown with different fluxes. The results with $\bar{F}_{0}=5 \times 10^{-5}$ (black), $5 \times 10^{-6}$ (blue), and $5 \times 10^{-7}$ (red) with the magnetic field are shown The red dashed lines shows the result with $\bar{F}_{0}=5 \times 10^{-7}$ without the magnetic field. The works are averaged over the horizontal plane. Each panel shows (a) $W_{\text {buo }}$, (b) $W_{\text {pre(h) }}$ (c) $W_{\operatorname{lor}(\mathrm{v})}$, and (d) $W_{\operatorname{lor}(\mathrm{h})}$. The definition of each work is shown in eq. (21)-(24). 
shows the horizontal pressure gradient work $\left(W_{\text {pre(h) }}\right)$. In most of the $\mathrm{CZ}$, the result is the same as with the energy flux, i.e., the work is always positive and accelerates the thermal convection. On the other hand, the acceleration at the base of the $\mathrm{CZ}$ is significantly depend on the energy flux. Smaller energy fluxes cause sudden increases of the horizontal pressure gradient work. When the flow strikes the stiff wall, this increases the horizontal pressure gradient and causes the horizontal flow. Stiff RZ behaves like a wall (we call it "wall effect"). Panel c shows the vertical Lorentz force work. The value is always negative which suppresses the thermal convection. This work does not depend on the energy flux in the CZ. Panel d shows the horizontal Lorentz force work. In the CZ, the work is moderately negative. The most important point is that the work shows a sudden decrease at the base of the $\mathrm{CZ}$ with the small energy flux. The negative Lorentz work means a gain of the magnetic energy. The increase of the horizontal velocity at the base of the $\mathrm{CZ}$ due to the wall effect increases the dynamo efficiency. This is the reason why the small energy flux causes the stronger magnetic energy at the bottom of the CZ. The horizontal magnetic field is preferentially amplified and the larger proxy of the inclination $\left(B_{\mathrm{h}} / B\right)$ is observed.

The dashed red lines in panels a and b of Fig. 13 shows the buoyancy and horizontal pressure works with $F_{0}=5 \times 10^{-7}$ without magnetic field. The significant increase of the buoyancy braking around the base of the CZ shows the importance of the magnetic field on the deceleration in the CZ. In magnetic case the horizontal pressure and the horizontal Lorentz work is almost balanced. In the case without magnetic field, the horizontal pressure work is significantly increased. This indicates that the magnetic field suppresses the horizontal velocity in the CZ. 


\section{Comparison with semi-analytic convection/overshoot model}

In order to understand our result properly, it is useful to compare our result with a semi-analytic convection/overshoot model. Rempel (2004) investigates the overshoot feature with several free parameters in broad range. It is explained that the overshoot characters are mainly determined with the imposed energy flux $\bar{F}_{0}$, the filling factor of the downflow $f$, and the mixing parameter between up- and downflow $\alpha_{\mathrm{d}}$. The energy flux and the filling factor are reduced to a single parameter $\Phi=\bar{F}_{0} / f$, which is important to determine the overshoot. We note that our normalization of the energy flux is a little different from his expression, which is $\bar{F}_{0}=F_{0} / p_{\mathrm{b}}\left(p_{\mathrm{b}} / \rho_{\mathrm{b}}\right)^{1 / 2}$. When $\Phi$ is large $\left(>10^{-2}\right)$, the overshoot shows subadiabatic penetration. On the other hand, small $\Phi$ shows almost adiabatic penetration with a steep transition to the RZ. Our calculation shows filling factor of $\sim 0.4$ in the downflow. Thus we estimate $\Phi=1 \times 10^{-6}$ in our calculation with the smallest energy flux. Our calculations show almost adiabatic propagation in the subadiabatic CZ with a steep overshoot of $d_{\mathrm{os}} / H_{\mathrm{b}} \sim 6.1 \bar{F}_{0}^{0.31}$. Rempel (2004) shows similar relation for transition region, in which there is steep transition from the adiabatic stratification (overshoot region) to the RZ. The obtained relation in Rempel (2004) is $d_{\mathrm{tr}} \sim 5\left(v_{\mathrm{c}} / c_{\mathrm{s}}\right) \sim 5 \Phi^{1 / 3}$. Here the filling factor does not change in our calculations and is the order of unity. Thus we conclude that the transition region in Rempel (2004) corresponds to our overshoot region. Slight change in the power low index between our numerical model (0.31) and the semi-analytical model $(1 / 3)$ could be explained with the insufficient resolution for the thin overshoot region in our calculation. The thickness of the overshoot region is not numerically converged and it is expected that higher resolution shows thinner overshoot with the smallest energy flux (see Fig. 6).

Rempel (2004) also suggests that the overshoot depth is mainly determined by the mixing length parameter $\left(\alpha_{\mathrm{d}}\right)$, where the mixing length $l$ is expressed as $l \sim \alpha_{\mathrm{d}} H_{\mathrm{b}}$. Smaller 
$\alpha_{\mathrm{d}}$ shows shorter overshoot depth (see Fig. 5 of Rempel (2004)). In our calculation, we hardly see adiabatic penetration below the $\mathrm{CZ}$, this indicates a very small $\alpha_{\mathrm{d}}$. Strong horizontal flow is caused around the base of the CZ, this significantly increase the mixing between up- and downflow in thin layer around the base of the CZ, this means a small $\alpha_{\mathrm{d}}$. Fig. 5 shows that the thickness of the strong horizontal flow around the base of the CZ is about $0.2 H_{\mathrm{b}}$, this efficiently suppresses the downflow in the CZ. This is seen in the panel a of Fig. 13 which shows significant suppression of the thermal convection around the base of the CZ. In the summary, we conclude that our calculation is consistent with the model in Rempel (2004) with $\Phi=10^{-6}$ and small mixing parameter $\alpha_{\mathrm{d}}(\sim 0.2)$. As a result the adiabatic penetration in the overshoot region is almost disappeared and the depth of the overshoot region in this paper corresponds to the transition region in Rempel (2004).

We also note that small $\alpha_{\mathrm{d}}$ causes small subadiabatic region in the $\mathrm{CZ}$. In our calculation the subadiabatic region is extended. This implies $\alpha_{\mathrm{d}}$ is large in the CZ. Small $\alpha_{\mathrm{d}}$ around the base of the $\mathrm{CZ}$ is caused by the sudden suppression of the vertical motion due to the stiff RZ. Thus $\alpha_{\mathrm{d}}$ can be dependent on space and larger in the middle of the CZ, which causes extended subadiabatic region.

\section{Summary and Discussion}

We have carried out a series of high-resolution calculations of the convection zone (CZ) and the radiation zone (RZ). The main target of this study is the interface region between the $\mathrm{CZ}$ and the RZ, i.e., the overshoot region (OS). The difficulty in the investigation of the solar OS is caused by the small normalized energy flux $\left(\overline{F_{0}}=F_{0} /\left(\rho_{\mathrm{b}} c_{\mathrm{b}}^{3}\right)\right)$. The solar value is $\bar{F}_{0} \sim 5 \times 10^{-11}$ at the base of solar CZ. We achieve unprecedentedly small value of the normalized energy flux $\left(\bar{F}_{0}=5 \times 10^{-7}\right)$ with keeping the resolution. Most compressible

calculations are carried out with $\bar{F}_{0} \sim 10^{-3}$ in order to avoid severe constraints of the sound 
wave in the CFL condition (an exception is Käpylä et al. 2007, with $\bar{F}_{0} \sim 10^{-5}$ ). We also investigated dependence of the small-scale dynamo on the energy flux, i.e., the stiffness of the RZ. Our main conclusions are:

1. The overshoot depth $\left(d_{\mathrm{os}}\right)$ scales with the energy flux $\left(\bar{F}_{0}\right)$ as $d_{\mathrm{os}} / H_{\mathrm{b}}=6.1 \bar{F}_{0}^{0.31}$, where $H_{\mathrm{b}}$ is the local pressure scale height. We estimate that the real solar overshoot depth is $0.004 H_{\mathrm{b}} \sim 250 \mathrm{~km}$.

2. Subadiabatic region is created even in the $\mathrm{CZ}$ with similar absolute value of the superadiabaticity in the superadiabatic CZ.

3. Essential deceleration is done in the subadiabatic CZ.

4. Smaller energy flux, i.e., stiffer RZ makes the small-scale dynamo more efficient and the magnetic field more horizontal with sudden increase of the horizontal pressure gradient around the base of the $\mathrm{CZ}$.

Our findings of the structures of the $\mathrm{CZ}$ and the $\mathrm{RZ}$ are summarized in Fig. 14.

Although we cannot directly address the issue about the large-scale dynamo and magnetic field in this study, we can suggest some implications. The subadiabatic layer at the bottom half of the $\mathrm{CZ}$ would be a good place to storage large magnetic flux. The downflow in the top half of the CZ efficiently transports the large-scale magnetic flux downward. This downflow is decelerated by the subadiabatic layer in the $\mathrm{CZ}$ and the large-scale magnetic field is less disturbed by the downflow.

Brummell et al. (2002) suggest that the rotation effect decreases the depth of the overshoot layer, since the inclination of the downflow is changed. It is expected that the rotation works almost the same manner even in high resolution and small energy flux achieved in this study. In addition, the rotation can generates the large-scale magnetic field. 


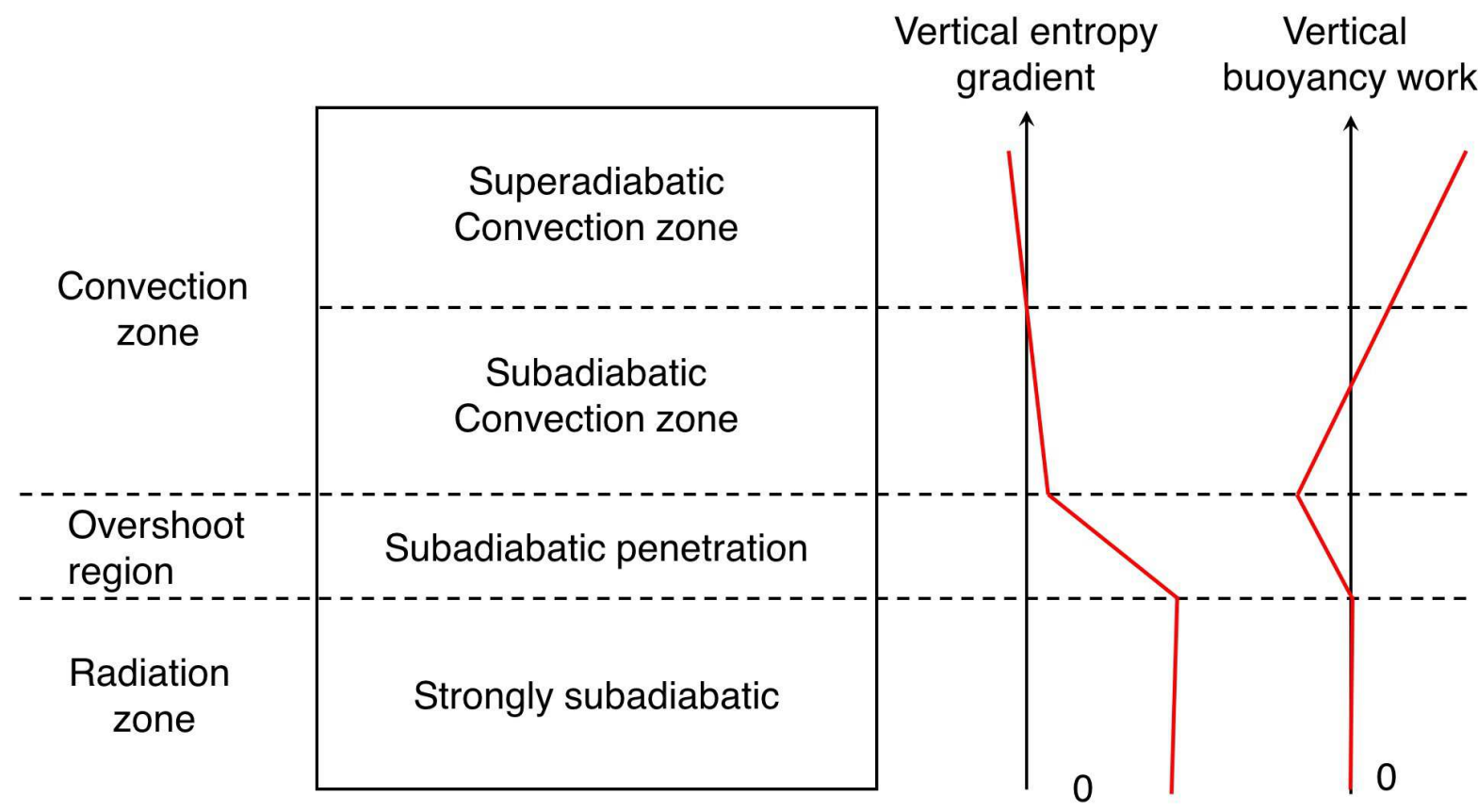

Fig. 14.- A schematic the CZ, the OS and the RZ describing the vertical entropy gradient $d s / d x$ and the vertical buoyancy work $-\rho g v_{x}-\partial p / \partial x v_{x}$. The quantities are plotted on horizontal axis with increasing toward the right. 
The large-scale coherent magnetic field efficiently suppresses the downflow and decreases the depth of the OS. In summary, the rotation effect tends to decrease the depth of the OS region. This should be investigated in the future research.

The absolute value of the superadiabaticity in the subadiabatic CZ is very small which is similar to that in superadiabatic $\mathrm{CZ}\left(|\delta| \sim \bar{F}_{0}^{2 / 3}\right)$. Using our result, the expected superadiabaticity around the bottom of the real solar CZ is $\delta=-1 \times 10^{-7}$ (subadiabatic). This is very unlikely to have an observable influence on the frequency of the acoustic modes, i.e., will be very difficult to detect with helioseismology.

Helioseismology has set the upper limit of the overshoot depth of $0.05 H_{p}$. On the other hand, typical simplified models underestimate or even ignore the effect of the subadiabatic $\mathrm{CZ}$ which has a main role to suppress the downflow. The overshoot below the $\mathrm{CZ}$ is overestimated $\left(\sim 0.5 H_{p}\right)$. This would be the discrepancy between the helioseismology and the simplified models.

Small density contrast between the bottom and top boundaries $\left(\rho_{\mathrm{b}} / \rho_{\text {top }} \sim 7\right)$ are used in this study, where $\rho_{\text {top }}$ is the density at the top boundary. The real sun has a density contrast of $10^{6}$. We note several possible influence of the higher density contrast on the overshoot. Generally it is expected that if the downflow is coherent from the solar surface (photosphere) to the bottom of the CZ, the filling factor of the downflow around the base of CZ becomes very small. Our previous study, however, with density contrast of $\sim 600$ also shows the filling factor of 0.4 as shown in this study (Hotta et al. 2014). This indicates that the instability around the donwflow is effective and donwflows cannot be coherent thoughout the entire CZ. Thus regarding the filling factor, our result could be reliable. Next, the higher density contrast tends to increase the convection velocity, this likely increases the overshoot depth. We are possibly underestimating the overshoot depth a little in this study due to small density contrast. 
We note that a recent helioseismic investigation suggests a smooth transition from the CZ to the RZ (Christensen-Dalsgaard et al. 2011). Rempel (2004) shows that smooth transition is possible only when the filling factor of the downflow is tiny and Mach number is large $(\mathrm{Ma}>0.1)$ even around the baser of the $\mathrm{CZ}$ or the deceleration is not efficient at the overshoot region due to low correlation between velocity and temperature (Xiong \& Deng 2001), which is not realized in our study. The tiny filling factor is achieved only when the downflow is coherent throughout the $\mathrm{CZ}$ with very large density contrast from the photosphere to the base of the CZ. This would be achievable with an unknown mechanism to suppress the instability around the downflow. This is a challenging issue to explore with numerical calculations.

As explained in the introduction, it is difficult to use the anelastic approximation and the reduced speed of sound technique for investigations of the overshoot region, since these cannot avoid the high Brunt-Väisälä frequency. They are, however, necessary to cover long time scale large-scale dynamo calculations. Thanks to the high stiffness of the solar RZ, the impenetrate boundary with the horizontal magnetic field at the base of the CZ nicely mimics the solar situation with a slight overestimation of the magnetic strength and inclination of the magnetic field. Softer RZ realized with large energy flux or small entropy gradient should not be used, since this significantly overestimate the storage of the large-scale magnetic field.

The author would like thank anonymous referee for his/her great suggestion which significantly improve the manuscript. The author is grateful to M. Rempel, H. Iijima, R. Cameron for their helpful comments on the manuscript. The results are obtained by using the K computer at the RIKEN Advanced Institute for Computational Science (Proposal number hp170239, hp170012, hp160026, hp160252, ra000008). This work was supported by MEXT/JSPS KAKENHI Grant Number JP16K17655, JP16H01169. This research was 
supported by MEXT as "Exploratory Challenge on Post-K computer" (Elucidation of the Birth of Exoplanets [Second Earth] and the Environmental Variations of Planets in the Solar System) 


\section{REFERENCES}

Basu, S. 1997, MNRAS, 288, 572

—. 2016, Living Reviews in Solar Physics, 13, 2

Basu, S., Antia, H. M., \& Narasimha, D. 1994, MNRAS, 267, 209

Browning, M. K., Miesch, M. S., Brun, A. S., \& Toomre, J. 2006, ApJ, 648, L157

Brummell, N. H., Clune, T. L., \& Toomre, J. 2002, ApJ, 570, 825

Brun, A. S., Miesch, M. S., \& Toomre, J. 2011, ApJ, 742, 79

Christensen-Dalsgaard, J., Monteiro, M. J. P. F. G., Rempel, M., \& Thompson, M. J. 2011, MNRAS, 414, 1158

Christensen-Dalsgaard, J., et al. 1996, Science, 272, 1286

Guerrero, G., Smolarkiewicz, P. K., de Gouveia Dal Pino, E. M., Kosovichev, A. G., \& Mansour, N. N. 2016, ApJ, 819, 104

Hotta, H., Rempel, M., \& Yokoyama, T. 2014, ApJ, 786, 24

-. 2015a, ApJ, 803, 42

-. 2015b, ApJ, 798, 51

—. 2016, Science, 351, 1427

Hotta, H., Rempel, M., Yokoyama, T., Iida, Y., \& Fan, Y. 2012, A\&A, 539, A30

Hurlburt, N. E., Toomre, J., Massaguer, J. M., \& Zahn, J.-P. 1994, ApJ, 421, 245

Käpylä, P. J., Korpi, M. J., Brandenburg, A., Mitra, D., \& Tavakol, R. 2010, Astronomische Nachrichten, 331, 73 
Käpylä, P. J., Korpi, M. J., Stix, M., \& Tuominen, I. 2007, in IAU Symposium, Vol. 239, Convection in Astrophysics, ed. F. Kupka, I. Roxburgh, \& K. L. Chan, 437-442

Masada, Y., Yamada, K., \& Kageyama, A. 2013, ApJ, 778, 11

Miesch, M. S., Elliott, J. R., Toomre, J., Clune, T. L., Glatzmaier, G. A., \& Gilman, P. A. 2000, ApJ, 532, 593

Monteiro, M. J. P. F. G., Christensen-Dalsgaard, J., \& Thompson, M. J. 1994, A\&A, 283, 247

Parker, E. N. 1993, ApJ, 408, 707

Pidatella, R. M., \& Stix, M. 1986, A\&A, 157, 338

Rempel, M. 2004, ApJ, 607, 1046

-. 2014, ApJ, 789, 132

Rempel, M., Schüssler, M., \& Knölker, M. 2009, ApJ, 691, 640

Roxburgh, I. W., \& Vorontsov, S. V. 1994, MNRAS, 268, 880

Roxburgh, L. W., \& Simmons, J. 1993, A\&A, 277, 93

Schmitt, J. H. M. M., Rosner, R., \& Bohn, H. U. 1984, ApJ, 282, 316

Singh, H. P., Roxburgh, I. W., \& Chan, K. L. 1995, A\&A, 295, 703

-. 1998, A\&A, 340, 178

van Ballegooijen, A. A. 1982, A\&A, 113, 99

Vitense, E. 1953, ZAp, 32, 135 
Vögler, A., Shelyag, S., Schüssler, M., Cattaneo, F., Emonet, T., \& Linde, T. 2005, A\&A, 429,335

Xiong, D. R., \& Deng, L. 2001, MNRAS, 327, 1137 